\title{
Temperature-dependent transport properties of a FeTe compound
}

\author{
PAVITRA DEVI LODHI ${ }^{1}$, NETRAM KAURAV ${ }^{1}, \mathrm{~K}^{\mathrm{K}}$ CHOUDHARY ${ }^{2, *}$ and Y K KUO ${ }^{3}$ \\ ${ }^{1}$ Department of Physics, Government Holkar Science College, Indore 452001, India \\ ${ }^{2}$ Army Cadet College, Indian Military Academy, Dehradun 248007, India \\ ${ }^{3}$ Department of Physics, National Dong Hwa University, Hualien 97401, Taiwan \\ *Author for correspondence (kkchoudhary1@yahoo.com)
}

MS received 26 November 2018; accepted 15 March 2019

\begin{abstract}
Temperature-dependent transport properties of a FeTe parent compound have been investigated by measurements of electrical resistivity $\rho(T)$, thermal conductivity $\kappa(T)$ and Seebeck coefficient $S(T)$. The sample was synthesized using a standard solid state reaction route via vacuum encapsulation and characterized by X-ray diffraction spectroscopy, which indicated a tetragonal phase with the space group $P 4 / \mathrm{nmm}$. The resistivity measurement data of the parent FeTe compound do not exhibit superconductivity however, it shows an anomaly in the temperature-dependent resistivity at around $67 \mathrm{~K}$, which corresponds to structural phase transition in the vicinity of a magnetic phase transition. In the low temperature regime, Seebeck coefficient, $S(T)$, exhibited an anomalous dip feature and negative throughout the temperature range, indicating an electron-like, single band charge carrier mechanism. We have also estimated the power factor and thermoelectric figure of merit $Z T$, which is found to be significantly smaller than other compositions of iron chalcogenides.
\end{abstract}

Keywords. Thermoelectric properties; thermal conductivity; resistivity; Seebeck coefficient.

\section{Introduction}

The discovery of superconductivity in iron chalcogenides $\mathrm{La}\left[\mathrm{O}_{1-x} \mathrm{~F}_{x}\right] \mathrm{Fe} A$ s at critical temperature $T_{\mathrm{c}}=26 \mathrm{~K}[1]$ has attracted wide attention of researchers to elucidate the mechanism of superconductivity and to explore the normal state thermoelectric properties. Later on other crystal structures of layered-Fe compounds such as $\mathrm{Ba}_{1-x} \mathrm{~K}_{x} \mathrm{Fe}_{2} \mathrm{As}_{2}$ [2], $\mathrm{Li}_{1-x} \mathrm{FeAs}$ [3] and FeSe [4] have also been reported to be superconducting. All these compounds have tetragonal crystal structures which are a square planar sheet of $\mathrm{Fe}$ in a tetrahedral environment with $\mathrm{Fe}^{+2}$ valences. Though, $\mathrm{FeTe}$ has a tetragonal crystal structure which is similar to that of superconducting $\mathrm{FeSe}$, but it does not show any sign of superconductivity. The effects of chemical substitutions and hydrostatic pressure were studied, the $T_{\mathrm{c}}$ of FeSe is increased up to $15 \mathrm{~K}$ by a limited substitution of Te on the Se site and increased up to $27 \mathrm{~K}$ by applying a hydrostatic pressure of $1.48 \mathrm{GPa}$ [5].

$\mathrm{Fe}_{1+y} \mathrm{Te}$ exhibits a structural and magnetic transition from a tetragonal paramagnetic phase to an orthorhombic antiferromagnetic phase at around $65 \mathrm{~K}$. Above the structural and magnetic transition temperature, the resistivity of $\mathrm{Fe}_{1+y} \mathrm{Te}$ represents weak temperature dependence, near the transition temperature. The resistivity decreases sharply and a metallic phase occurs below the transition temperature [6]. A maximum magnitude of the Seebeck coefficient $S$ of 11 compound is observed in the vicinity of the structural/magnetic transition temperature, attributing to a change in the phonon scattering mechanism [7]. The Seebeck effect of the single crystal $\mathrm{Fe}_{1+y} \mathrm{Te}$ [7] shows a very similar curve measured in another single crystal [8] and polycrystalline samples [6] and corroborates with the transition observed in the resistivity curve. In comparison with the Seebeck coefficient of other Fe-based parent compounds, $\mathrm{Fe}_{1+y}$ Te shows very small values in the wide temperature range [9].

The effect of doping on the thermoelectric properties of $\mathrm{FeSe}_{1-y}$ samples is analysed [10]. The optimal doping enhances $S$ and suppresses resistivity $\rho$ at the same time, resulting in considerable enhancement in power factors (PFs) $S^{2} / \rho$ and figure of merit $(Z T)=S^{2} \sigma T / \kappa=S^{2} T / \rho \kappa$. The value of the $\mathrm{PF}$ is optimized to $15 \mu \mathrm{W} \mathrm{K} \mathrm{K}^{-2} \mathrm{~cm}^{-1}$ and $Z T$ up to $10^{-2}$ by $5 \%$ Co doping in FeSe [10]. Almost negligible temperature dependence of the Seebeck effect $S$ at high temperatures points to a more localized charge carriers in iron chalcogenides [9]. The study of the thermoelectric transport properties reveals precious information about charge carriers, density of states, Fermi surface and scattering mechanisms in iron chalcogenides. In present work, we report the synthesis, characterization and measurements of the thermoelectric transport properties in the FeTe compound, which was prepared by an inexpensive and yet simple method. The thermoelectric and transport properties such as thermal conductivity $(\kappa)$, Seebeck coefficient $(S)$, electrical resistivity $(\rho), \mathrm{PF}$ and thermoelectric $Z T$ of the FeTe parent compound were measured and analysed. 


\section{Experimental}

The bulk polycrystalline FeTe sample was synthesized through a standard solid state reaction route via vacuum encapsulation. The mixed powder was subsequently pelletized and then encapsulated in an evacuated $\left(10^{-3}\right.$ Torr $)$ quartz tube. The encapsulated tube was then heated at $750^{\circ} \mathrm{C}$ for $12 \mathrm{~h}$ and slowly cooled down to room temperature. The heating schedule was repeated couple of times with intermediate grinding. Phase identification of the powder sample was performed using a Rigaku Diffractometer with $\mathrm{CuK} \alpha$ radiation $(\lambda=1.54 \AA$ ) $)$. Resistivity and thermal conductivity measurements were carried out by standard four probe method in a helium-closed cycle refrigerator. The Seebeck coefficient measurement was carried out from 4 to $300 \mathrm{~K}$ using a direct heat pulse technique.

\section{Results and discussion}

The room temperature X-ray diffraction (XRD) pattern for the FeTe sample along with their Rietveld analysis is presented in figure 1. The structure of FeTe was refined with the tetragonal space group $P 4 / \mathrm{nmm}$ (space-group No. 129). Fe is situated at the coordinate position $(3 / 4,1 / 4,0)$ and Te at $(1 / 4,1 / 4, z)$ in FeTe compounds and the values obtained from Rietveld analysis are tabulated in table 1 . The peaks belonging to $\mathrm{FeTe}$ (ICDD No. 98-005-1191) were identified and also a small

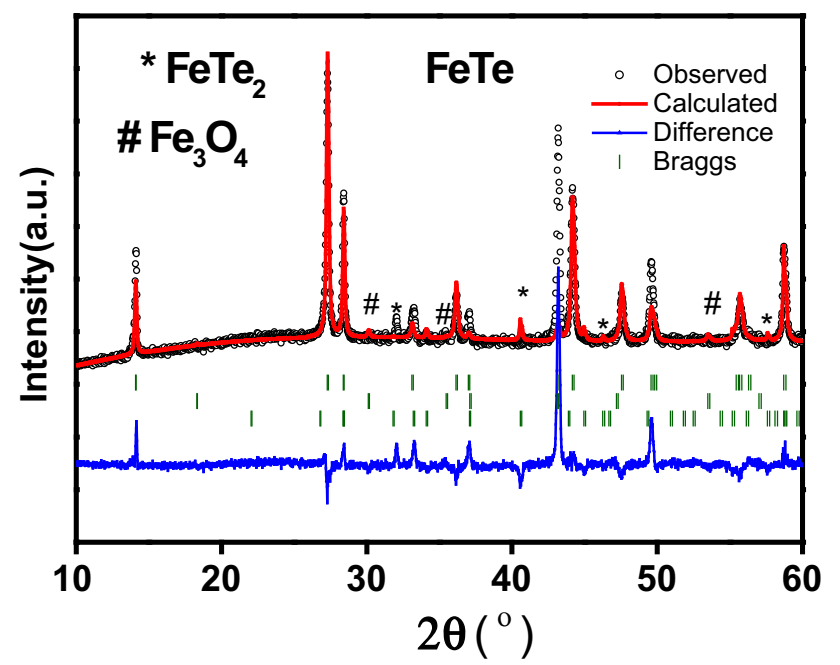

Figure 1. Observed room temperature XRD pattern (open circle) and Rietveld fit (red line) of the FeTe compound; the blue line shows difference of observed values and Rietveld fit values. trace of impurity phases of (\#) $\mathrm{Fe}_{3} \mathrm{O}_{4}$ and (*) $\mathrm{FeTe}_{2}$ was found in FeTe. The formation of $\mathrm{Fe}_{3} \mathrm{O}_{4}$ is expected due to powder handling in air and oxygen entrapped inside the quartz tube before sintering. The formation of $\mathrm{Fe}_{3} \mathrm{O}_{4}$ caused a diminution of iron concentration in the samples leading to the formation of the impurity phase of $\mathrm{FeTe}_{2}$ as can be seen in the XRD patterns.

The temperature-dependent resistivity $\rho(T)$ of the FeTe polycrystalline compound is shown in figure 2 . It can be seen that FeTe does not show superconductivity, unlike the other compositions of iron chalcogenides who show a superconducting transition in resistivity between 13 and $16 \mathrm{~K}$ [11]. Resistivity exhibits power temperature dependence at low temperatures below $67 \mathrm{~K}$ and takes a remarkable maximum near $67 \mathrm{~K}$, which corresponds to the anti-ferromagnetic and structural transitions observed in FeTe. On further increase in temperature resistivity decreases with a small change in the slope and exhibits semiconducting behaviour. At low temperatures, below antiferromagnetic transition temperature $\left(T_{\mathrm{N}}\right)$, the small but coherent quasi-particles show the metallic behaviour, which is estimated using the Bloch-Gruneisen (BG) function of temperature-dependent resistivity, by incorporating electron-phonon scattering mechanism [12]. Dashed line in figure 2 represents the BG fit to resistivity [12]. A small but sharp quasi-particle peak emerges in the bicollinear anti-ferromagnetic state of the FeTe compound. Such an incoherent spectral weight is responsible for the semiconducting behaviour of the resistivity at high temperatures. Resistivity in

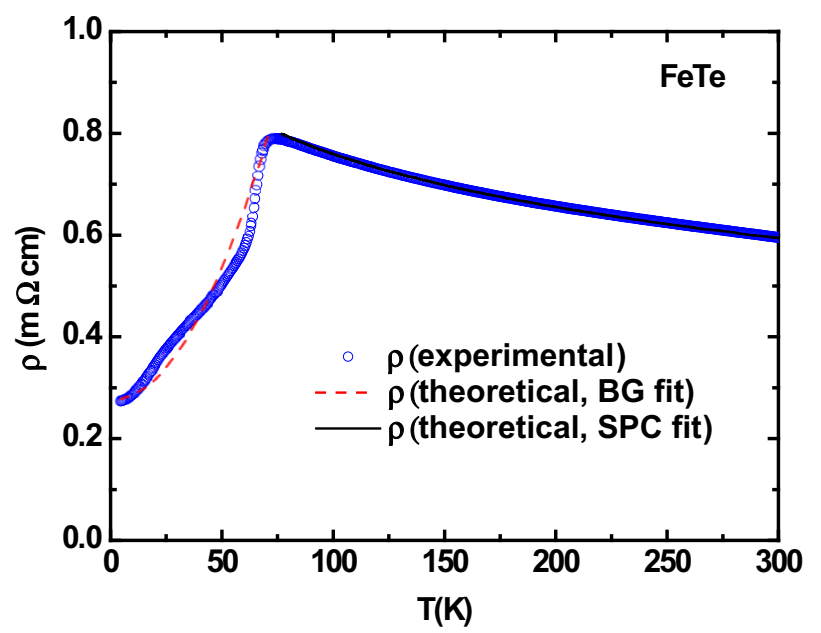

Figure 2. Variation of electrical resistivity $\rho(T)$ with temperature for the FeTe compound; the dashed line represents BG fit and the solid line represents SPC fit to resistivity.

Table 1. Structural parameter obtained from Rietveld refinement analysis of the FeTe sample.

\begin{tabular}{|c|c|c|c|c|c|c|c|c|c|c|}
\hline & $a(\AA)$ & $b(\AA)$ & $V\left(\AA^{3}\right)$ & $\% \mathrm{FeTe}$ & $\% \mathrm{FeTe}_{2}$ & $\% \mathrm{Fe}_{3} \mathrm{O}_{4}$ & $R_{\mathrm{wp}}(\%)$ & $R_{\mathrm{p}}(\%)$ & $R_{\exp }(\%)$ & $x^{2}$ \\
\hline $\mathrm{FeTe}$ & 3.823 & 6.286 & 91.859 & 94.62 & 3.77 & 1.67 & 47.8 & 48.7 & 21.94 & 10.2 \\
\hline
\end{tabular}




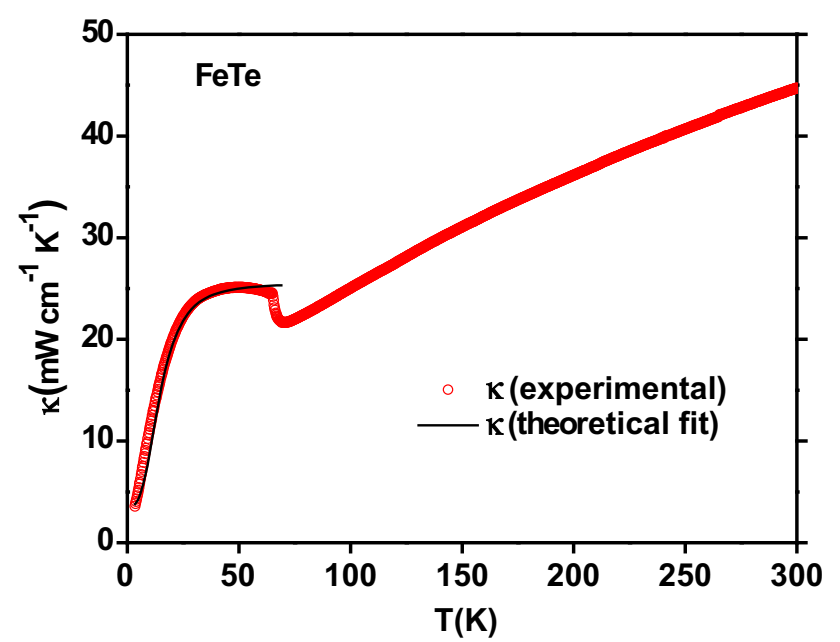

Figure 3. Variation of thermal conductivity $\kappa(T)$ as a function of temperature for $\mathrm{FeTe}$; open circles represent experimentally measured data of $\kappa$ whereas, the solid line represents theoretical fit using a phonon scattering mechanism.

the semiconducting phase, at higher temperatures $(T>67 \mathrm{~K})$ is described within a small polaron conduction (SPC) model [13]. The solid line in figure 2 represents the theoretical fit to resistivity using the SPC model. The electron-phonon scattering-based BG model together with the SPC model can explain the temperature-dependent resistivity behaviour in overall temperature ranges.

The thermal conductivity $(\kappa)$ measurement data of the FeTe compound are shown in figure 3 . At low temperatures, thermal conductivity $(\kappa)$ increases and a broad peak appears around $50 \mathrm{~K}$ in the FeTe sample (open circles in figure 3). This is a typical feature, representing the reduction in phonon scattering and the corresponding increase in the phonon mean free path in solids at low temperatures. The maxima take place at the temperature where the phonon mean free path is approximately equal to the crystal site distance attributing to the generalized phonon umklapp scattering process. The thermal conductivity $(\kappa)$ at low temperatures is theoretically estimated by the phonon scattering mechanism [14,15], considering scattering of phonons with defects, grain boundaries, electrons and phonons. The peak observed in $\kappa$ depends on competition among various operating scattering processes. The solid line in figure 3 represents the theoretical fit to $\kappa$.

Figure 4 shows the temperature-dependent Seebeck coefficient $S(T)$ curve for the FeTe sample. In the FeTe sample, $S(T)$ undergoes an abrupt step-like change at the magnetic/structural transition around $70 \mathrm{~K}$, which is consistent with the step change observed in the temperature-dependent resistivity curve. Further, $|S|$ reaches a maximum value and eventually tends to disappear when the temperature tends to zero. It is found to be negative throughout the temperature range, indicating an electron-like charge carrier conduction mechanism. The narrow-band Hubbard model could likely be a source of the complex $S(T)$ behaviour observed in the 11

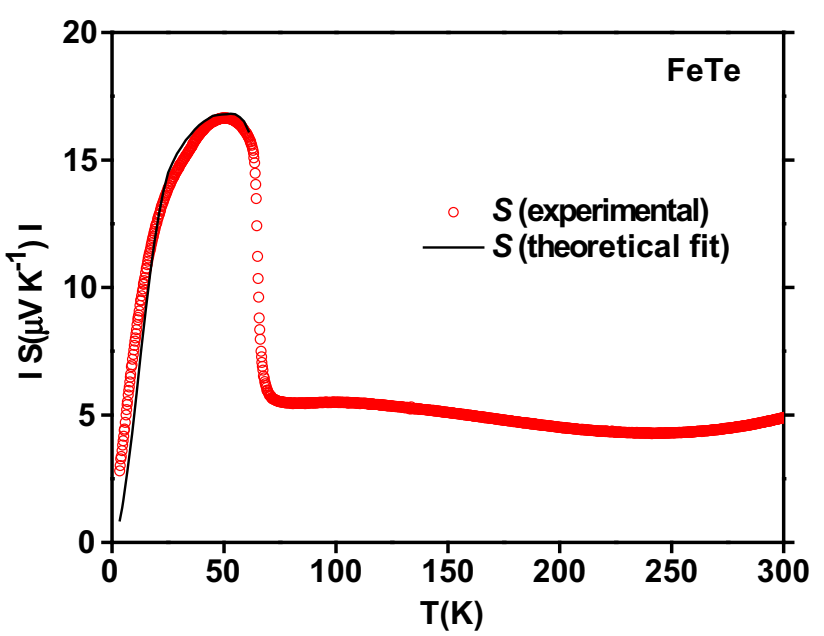

Figure 4. Variation of the Seebeck coefficient $(S)$ of FeTe as a function of temperature; experimentally measured $S$ is presented by open circles and the solid line represents theoretical fit to $S$.

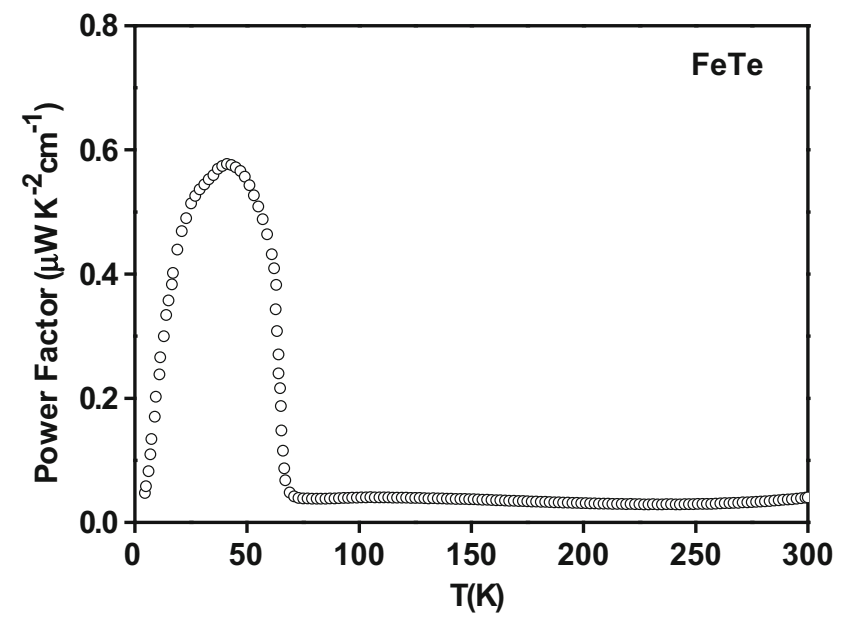

Figure 5. Variation of $\operatorname{PF}\left(S^{2} / \rho\right)$ with temperature.

type compounds. The Seebeck coefficient $(S)$ is determined by electron bands in the FeTe parent compound which is in contrast with other compositions of $\mathrm{FeTe}_{1-x} \mathrm{Se}_{x}$ single crystals [7] and rare earth-doped FeAsO compounds [16], where a multi-band effect is clearly observed. The temperature dependent $S(T)$ at low temperatures, below Debye temperatures $\left(\theta_{\mathrm{D}} \approx 200 \mathrm{~K}\right)$, is theoretically estimated considering phonon drag [14,15] and carrier diffusion [17] effects. The solid line in figure 4 represents the theoretical fit to $S(T)$.

In order to analyse the applicability of $\mathrm{FeTe}$ as an low temperature thermoelectric material, we estimated the temperature-dependent PFs $\left(S^{2} / \rho\right)$ and $Z T=S^{2} \sigma T / \kappa$ $=S^{2} T / \rho \kappa$. The PF is plotted as a function of temperature in figure 5 which attains the maximum value of $0.6 \mu \mathrm{W} \mathrm{K}^{-2} \mathrm{~cm}^{-1}$ at around $41 \mathrm{~K}$, just below the structural/magnetic transition temperature and sharply falls down to one tenth of this value at transition temperature. Variation 


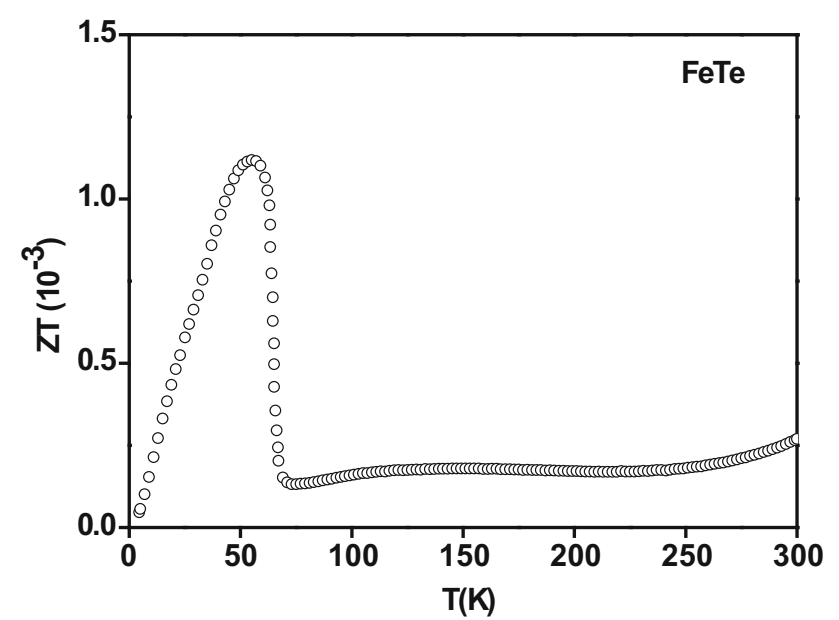

Figure 6. Variation of $Z T=\left(S^{2} T / \rho \kappa\right)$ with temperature.

in $Z T$ as a function of temperature is presented in figure 6 which exhibits maxima at $T=55 \mathrm{~K}$, attains a maximum value of $1.1 \times 10^{-3}$ and sharply falls down after structural transition temperature $T=63 \mathrm{~K}$. The magnitude of the $\mathrm{PF}$ and $Z T$ of FeTe are much smaller as compared to other compositions of $\mathrm{FeTe}_{1-x} \mathrm{Se}_{x}$ single crystals [7] and optimally Co-doped FeSe [10], which ruled out the applicability of this material as a low temperature thermoelectric material. However, this compound is still technologically important material being only composition having a dominated electron band in this series of compounds and emerged as a powerful electron-doped low temperature thermoelectric material.

\section{Conclusions}

The temperature-dependent thermoelectric and transport properties such as thermal conductivity $\kappa(T)$, Seebeck coefficient $S(T)$, electrical resistivity $\rho(T), \mathrm{PF}$ and thermoelectric $Z T$ of the FeTe parent compound have been investigated. Measurements of $\rho(T)$ exhibit that FeTe is a non-superconducting compound, however, it shows an anomaly in temperaturedependent resistivity at around $67 \mathrm{~K}$, which corroborates to the anti-ferromagnetic and structural transitions. Due to this structural/magnetic phase transition, FeTe shows a transition from metallic to semiconducting resistivity $\rho(T)$ behaviour at around $67 \mathrm{~K}$. The Seebeck coefficient $S(T)$ for the FeTe sample observed to be negative throughout the temperature range, indicating electron-like charge carriers and a single band mechanism. $S(T)$ and $\kappa(T)$ produce a broad peak at the magnetic/structural transition, around $67 \mathrm{~K}$, and sharply decreases on an increase in temperature. Due to this peculiar temperature dependence of transport properties, the PF and thermoelectric
$Z T$ also show a peak at around 41 and $55 \mathrm{~K}$, respectively and sharply fall down on further increase in temperature. The maximum values of $\mathrm{PF}=0.6 \mu \mathrm{W} \mathrm{K} \mathrm{K}^{-2} \mathrm{~cm}^{-1}$ and $Z T=1.1 \times 10^{-3}$ have been obtained in the FeTe compound which are comparatively smaller than other compositions of optimally-doped iron based superconductors.

\section{Acknowledgements}

Dr V P S Awana, Superconductivity and Cryogenics Division, CSIR-National Physical Laboratory, New Delhi, is gratefully acknowledged for providing the sample preparation facilities. Financial support from the UGC-DAE Centre for Scientific Research, Indore under CRS projects (NK) and the Ministry of Science and Technology of Taiwan under Grant Nos. MOST-103-2112-M-259-008-MY3 and MOST-106-2112-M-312 259-002-MY3 (YKK) are gratefully acknowledged.

\section{References}

[1] Kamihara Y, Watanabe T, Hirano M and Hosono H 2008 J. Am. Chem. Soc. 1303296

[2] Rotter M, Tegel M and Johrendt D 2008 Phys. Rev. Lett. 101 107006

[3] Wang X C, Liu Q, Lv Y, Gao W, Yang L X, Yu R C et al 2008 Solid. State Commun. 148538

[4] Hsu F C, Luo J Y, Yeh K W, Chen T K, Huang T W, Wu P M et al 2008 Proc. Natl. Acad. Sci. USA 10514262

[5] Mizuguchi Y, Tomioka F, Tsuda S and Yamaguchi T 2008 Appl. Phys. Lett. 9352505

[6] Tropeano M, Pallecchi I, Cimberle M R, Ferdeghini C, Lamura G, Vignolo M et al 2010 Supercond. Sci. Technol. 23054001

[7] Pallecchi I, Lamura G, Tropeano M, Putti M, Viennois R, Giannini E et al 2009 Phys. Rev. B 80214511

[8] Matusiak M, Pomjakushina E and Conder K 2012 Physica $C 48321$

[9] Pallecchi I, Caglieris F and Putti M 2016 Supercond. Sci. Technol. 29073002

[10] Thomas E L, Wong-Ng W, Phelan D and Millican J N 2009 J. Appl. Phys. 105073906

[11] Mizuguchi Y and Takano Y 2010 J. Phys. Soc. Jpn. 79102001

[12] Ziman J M 1960 Electrons and phonon (New York: Oxford University Press)

[13] Shaikh M W, Varshney D and Mansuri I 2009 J. Alloys Compd. 486726

[14] Callaway J 1991 Quantum theory of the solid state (London: Academic Press)

[15] Barnard R D 1972 Thermoelectricity in metals and alloys (London: Taylor and Francis Ltd.)

[16] Caglieris F, Braggio A, Pallecchi I, Provino A, Pani M, Lamura G et al 2014 Phys. Rev. B 90134421

[17] Inabe T, Ogata H, Maruyama Y, Achiba Y, Suzuki S, Kikuchi K et al 1992 Phys. Rev. Lett. 693797 